\title{
Prognostic Markers in Advanced Heart Failure
}

\author{
Parth Pramod Godhiwala ${ }^{1}$, Sourya Acharya ${ }^{2}$, Sunil Kumar ${ }^{3}$, Charan Bagga ${ }^{4}$ \\ 1, 2, 3, 4 Department of Medicine, Datta Meghe Institute of Medical Sciences \\ (Deemed to Be University), Sawangi (Meghe), Wardha, Maharashtra, India.
}

\section{ABSTRACT}

Heart Failure is a crippling condition with a very poor outcome. Advanced heart failure is the endpoint in patients of heart failure. Mortality rates are significantly higher among these patients. Heart failure is an impairing condition with a poor prognosis, especially during the advanced stage when patients progress with shorter survival rates and mortality rates higher than $50 \%$ during the first year of follow-up. Early identification of those patients who might experience the worst progression or premature death is vital, as this would allow intervention in an attempt to change the natural course of their disease. Conventionally, in evaluating heart failure populations, the magnitude of clinical manifestations has proved to be a valuable tool for prognosis stratification. Because of their symptoms, these patients need hospitalization and form a poor prognosis population, although tests may help to identify which ones will have the worst clinical progression.

Various tests and risk scores are therefore developed as prognostic markers in advanced heart failure. Early diagnosis and treatment can help change the natural course of the disease. Commonly used prognostic markers include HbA1c, eGFR, LVEF, 6MWT, and NT-proBNP. These markers can help predict mortality in these patients. Before the use of these markers, the prognosis was defined by clinical presentation and severity of symptoms but it was not possible to decide as to which patient will have the worst outcome based only on symptoms. This review is an attempt to summarize the basic knowledge of various prognostic markers mentioned above used in advanced heart failure.

\section{KEY WORDS}

Advanced Heart Failure, Prognosis, 6MWT, eGFR, LVEF, NT-proBNP, HbA1c
Corresponding Author: Dr. Sourya Acharya,

Professor,

Department of Medicine,

Datta Meghe Institute of Medical Sciences

(Deemed to Be University),

Sawangi (Meghe), Wardha,

Maharashtra, India.

E-mail: souryaacharya74@gmail.com

DOI: $10.14260 /$ jemds $/ 2021 / 8$

How to Cite This Article:

Godhiwala PP, Acharya S, Kumar S, et al. Prognostic markers in advanced heart failure. J Evolution Med Dent Sci 2021;10 (01):39-44, DOI: 10.14260/jemds/2021/8

Submission 08-08-2020,

Peer Review 14-10-2020,

Acceptance 20-10-2020,

Published 04-01-2021.

Copyright (C) 2021 Parth Pramod Godhiwala et al. This is an open access article distributed under Creative Commons Attribution License [Attribution 4.0 International (CC BY 4.0)] 


\section{BACKGROUND}

Heart failure is a "complex clinical syndrome resulting from impairment of ventricular filling or ejection of blood associated with symptoms of dyspnoea, fatigue, and

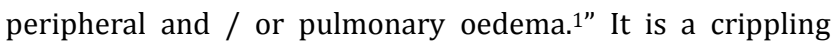
condition with a very poor prognosis. Morbidity and mortality rates are nearly $50 \%$ after one year of follow-up. Early identification of risk factors and timely made interventions are two important aspects of changing the natural course of the disease. ${ }^{2}$ Heart failure is rightly called an "epidemic". Its epidemiology is very complicated as multiple factors interact in a complex manner which has a big impact on its prevalence. ${ }^{3}$ Advanced heart failure is a subset of patients who are refractory to medical line of management as per the guidelines. Most of the heart failure-related hospitalisations and deaths are seen in these patients. There is an increase in the prevalence of advanced HF due to improvement in treatment protocols. However, quality of life continues to remain poor for this subgroup of HF. ${ }^{4}$

The diagnosis of HF is mainly a clinical one. There is no such feature that can help differentiate between HF and advanced HF. Multiple modalities are used for their diagnosis. These include clinical, functional, haemodynamic, imaging, and biomarker data. The presence of multiple parameters associated should raise the possibility of stage D HF. Hence, for this reason, risk scores were developed. They help facilitate the diagnosis and prognosis of advanced HF. ${ }^{4}$ Advanced heart failure is most commonly caused by Coronary Artery Disease (CAD). The second most common cause is cardiomyopathies while rheumatic heart disease is third in the list. ${ }^{5}$ Recently, many variables have been used for defining prognosis in patients with advanced HF. ${ }^{2}$ Many articles have used 6MWT, LVEF, NT-proBNP, eGFR, and HbA1c as prognostic markers. Hereby, this article highlights in brief about these markers and their use as markers in the modern world.

\section{EPIDEMIOLOGY}

The overall prevalence rate of HF in the USA is $1.76 \%$ which is approximately 4.8 million individuals of the total population. The prevalence is $2 \%$ among people age between $40-59$ years. It rises to $5 \%$ in the age group $60-69$ years which further rises to $10 \%$ in people age more than 70 years. ${ }^{6}$ When the above numbers are projected to the Indian population, the approximate people affected by $\mathrm{HF}$ would be around 10 million which constitutes about $0.9 \%$ of the total Indian population. ${ }^{6}$ It is estimated that the prevalence of stage D HF ranges from $6 \%$ to $25 \%$ of the heart failure cases. ${ }^{4}$ The largest available registry to date of patients with chronic advanced HF is Acute Decompensated Heart Failure National Registry LM (ADHERE LM). According to the registry, approximately $5 \%$ of patients suffering from HF have Stage D disease. These patients are refractory to the guideline-based line of therapy. ${ }^{7}$

\section{DEFINITION}

According to the heart failure guidelines by American Heart Association (AHA) / American College of Cardiology (ACC)
(2013) "Heart failure is a complex clinical syndrome that results from any structural or functional impairment of ventricular filling or ejection of blood." The most common manifestations of heart failure are dyspnoea and fatigue often leading to limiting exercise tolerance, and fluid retention, which eventually leads to pulmonary and / or peripheral oedema. ${ }^{1}$

Advanced HF is defined by the Heart Failure Society of America as "The presence of progressive and / or persistent severe signs and symptoms of heart failure despite optimized medical, surgical, and device therapy. It is generally accompanied by frequent hospitalization, severely limited exertional tolerance and poor quality of life, and is associated with high morbidity and mortality. Importantly, the progressive decline should be primarily driven by the heart failure syndrome. ." $^{\text {" }}$

\section{PROGNOSTIC MARKERS}

The severity of the disease is defined by many parameters. The use of these parameters in today's world is the result of many clinical trials that have happened worldwide in the past. They also define the prognosis of heart failure.

\section{LVEF}

Left Ventricular Ejection Fraction (LVEF), is one such marker. It shows the extent of the structural and functional abnormalities of the ventricle. LVEF is commonly used as a guide to explain the poor prognosis, morbidity, and mortality in HF. ${ }^{9}$ A low EF (Ejection Fraction) $(<20 \%)$ is conventionally viewed as a gross functional abnormality. It is usually associated with a dilated LV (Left Ventricle) chamber which is identified as a structural abnormality. ${ }^{9}$ The simultaneous presence of diastolic dysfunction decreases survival rates among patients with HF.

A lot many studies recently have confirmed the prognostic impact of change in LVEF in patients with HF with reduced EF (HFrEF). It is now proven that the recovery of LVEF, known as 'recovered EF', when occurs in HFrEF (Heart Failure with Preserved Ejection Failure) patients is associated with improved prognosis. ${ }^{10}$

Maylene Wong et al. studied the relationship between LVEF and outcome in 5010 patients enrolled in the Val-HeFT trial. Baseline echocardiograms were obtained for all patients on admission. At the end of 23 months, an echocardiogram was repeated for all patients. Decreasing LVEF was associated with poor prognosis and increased mortality. First quartile patients had a significantly lower mortality rate with a mean LVEF 35 $\%$ than those in the fourth quartile with a mean LVEF of $17 \%$. Mortality rates were $14 \%$ and $26 \%$ respectively. LVEF lower than $20 \%$ were invariably associated with poor survival. ${ }^{11}$

Maria R. Costanzo et al. did an analytical study well-known as the ADHERE LM study to explain the features of advanced HF. They studied data from 1433 patients during the entire study period. They found that mean LVEF was $29.5 \pm 14.1 \mathrm{in}$ the ADHERE LM registry. ${ }^{7}$

Carolyn S.P. Lam et al. studied ethnic and regional differences among HF patients in the Asian population. Mean LVEF was calculated as 30 (range: 24 - 35), 29 (range: 25 - 33) and 25 (range: 20 - 31) in Northeast Asia $(n=1658)$, South Asia 
$(\mathrm{n}=1436)$ and Southeast Asia $(\mathrm{n}=2182)$ respectively. Overall mean LVEF was 28 (range: 22 - 33) in total 5276 subjects. ${ }^{12}$

\section{2. eGFR (Estimated Glomerular Filtration Rate)}

A number of studies have discussed a connection between impaired renal function and poor outcomes in acute as well as chronic HF. Drugs used in the treatment of HF can itself affect renal functioning in many ways leading to decreased Glomerular Filtration Rate (GFR). It is an indication of poor prognosis. ${ }^{13}$

Mortality rates are very high in patients with reduced eGFR or reducing eGFR. This has seen observed in two landmark RCTs (Randomized Controlled Trials) that analyzed the data retrospectively. ${ }^{14,15}$ Multiple factors are considered to cause renal dysfunction, out of which venous congestion and reduced renal perfusion most common and important factors. These two factors are primarily influenced by compromised cardiac functioning. 16

Testani et al in their study on heart failure patients observed early reduction in GFR is associated with high mortality rates. This landmark study known as the SOLVD (Studies of Left Ventricular Dysfunction) study, showed higher mortality in the placebo group. The Enalapril group did not show a reduction in GFR. As Testani et al concluded that "(1) reduced GFR directly contributes to worse outcomes and (2) reduced GFR is a marker of worsening HF and, thereby, is related with poorer outcomes. ${ }^{17}$

V.K. Chopra et al. had conducted a study over a period of 4 years (2014 - 2017) in 5590 subjects. The mean eGFR in their study was $76.1 \pm 27.7$. They also had studied mean eGFR among mortality and non-mortality group. Mean eGFR in the mortality group was $68.5 \pm 30.2$ and in the non-mortality group was $77.7 \pm 26.9 .18$

Pooja Dewan et al. had studied mean eGFR in 13174 patients of HFrEF. A comparison of patient clinical features and outcomes between Asia, the Americas, and Europe and also within Asia was done by them. They found out that mean eGFR was $65.8 \pm 19.1$ in Western Europe $(\mathrm{n}=3521), 70.0 \pm$ 19.5 in Central / Eastern Europe $(\mathrm{n}=4758), 61.7 \pm 17.7$ in North America $(\mathrm{n}=613), 77.5 \pm 29.5$ in India $(\mathrm{n}=1390)$ and $80.4 \pm 21.0$ in China $(\mathrm{n}=833) \cdot{ }^{19}$

\section{Hyponatremia}

Hyponatremia defined as "a serum sodium concentration < $135 \mathrm{mmol} / \mathrm{L}$," is a very familiar finding in HF. In the OPTIMECHF study, it was observed in $27 \%$ of the total study population. ${ }^{20}$ Hyponatremia has proved its worth as a predictor of poor outcomes in patients admitted for acute heart failure in this study. ${ }^{21}$

In ACTIV in CHF trial, low sodium levels were present in 21 $\%$ of patients hospitalized as a case of acute decompensated heart failure. ${ }^{22}$

In patients with chronic HF, serum sodium levels are wellknown predictors of mortality. ${ }^{23}$ While comparing serum sodium levels with mortality, and inversely proportional relation was documented. In-hospital and sixty-day mortality rates, both were significantly higher for patients with low onadmission sodium levels. It was also observed that mortality rates reduced by $25 \%$ with an increase in serum sodium by 5 meq / l on 60-days follow-up. ${ }^{21}$
Mihai Gheorghiade et al. performed a study on 433 hospitalized patients diagnosed to have heart failure. This was done to estimate the efficacy of a pulmonary artery catheter plus clinical assessment vs clinical assessment alone in managing treatment protocols in patients hospitalized with NYHA class IV HF in patients with LVEF $<30 \%$. This research is well-known as The ESCAPE trial. It was observed that mild hyponatraemia was invariably present in patients with HF. A persistently lower level of serum sodium is an independent mortality predictor of $\mathrm{HF}$ hospitalisation, and rehospitalisation or in-hospital death. ${ }^{24}$

Giovanni Corona et al. performed a meta-analysis to see the impact of a reduction in serum sodium levels on mortality in HF. They concluded that a moderate reduction in sodium levels is related to increased mortality rates. ${ }^{25}$

Asim A Mohammed et al. conducted a study to observe a relation between natriuretic peptides, hyponatremia, and outcomes in acutely decompensated heart failure. They concluded that reduced serum sodium levels are directly related to adverse outcomes in acute decompensated HF. Mortality rates are very high in patients having a co-existence of hypernatremia and raised Nt-proBNP. ${ }^{26}$

\section{Six-Minute Walk Test (6-MWT)}

6-MWT is a commonly used exercise test to evaluate the functional capacity of an individual. ${ }^{27}$ It can also be used to check the therapeutic effects of interventions. ${ }^{28}$ In patients with HF who are enrolled for Cardiac Rehabilitation (CR) programs, 6-MWT is done pre-rehabilitation and postrehabilitation to compare the distances walked. An improvement in the distance covered is a good indicator. Failing to do so results in high mortality. ${ }^{29} \mathrm{~A}$ cut-off value of $300(\mathrm{~m})$ is set as a comparison level. Any patient who covers a distance of less than $300 \mathrm{~m}$ has a poor prognosis. 6-MWT is a reliable, cheap, and easy to conduct prognostic marker of mortality in HF. 6-MWT is influenced by factors like age, stature, and BMI. Hence, while interpreting the results of the 6-MWT in patients with HF, the above-mentioned factors must be considered. ${ }^{30}$

The 6-minute walk test distance can be used for cardiovascular mortality risk stratification. Various cut-off values are used as the prognostic value in HF patients based upon the clinical characteristics. In 2 studies conducted in past, a distance of $<200$ to $220 \mathrm{~m}$ was set as a cut-off point that related with increased risk of mortality in refractory HF and NYHA Class III and IV patients, ${ }^{31}$ while in patients with the milder form of heart failure (NYHA Class II), a distance $<520$ $\mathrm{m}$ at 18-month follow-up was associated with increased cardiovascular mortality risk. ${ }^{32}$

Kinga Wegrzynowska-Teodorczyk et al. conducted an observational study to measure the distance covered during a 6-MWT and its use in predicting hospitalisation rates and longterm mortality in male patients with systolic HF. They concluded that a short distance covered during the 6-MWT is a threatening sign in patients with HF. The distance covered was directly related to the stage of HF. It showed its implication as prognostic marker during the one-year and the three-year follow-up. They also observed that a shorter distance in the 6-MWT was associated with high plasma NTpro BNP levels and increased uric acid levels. The hospitalisation rate and mortality rate, both were on the higher side during the one-year and three-year follow-up. ${ }^{33}$ 
To assess the role of the 6MWT in the evaluation of patients with advanced HF, Lawrence P. Cahalin et al had performed a study in 45 patients. Mean 6MWT distance ambulated was $310 \pm 100 \mathrm{~m}$. 6MWT distance less than $300 \mathrm{~m}$ was associated with increased mortality rate or pre-transplant repeated hospital admission within 6 months $(p=0.04) \cdot{ }^{34}$

Monica R. Shah et al had performed a study to study the prognostic usefulness of the 6-MWT in patients with advanced HF due to cardiomyopathy (ischemic or non-ischemic). The study was performed on 440 patients. They had divided patients into 2 groups: able to walk $(\mathrm{n}=365)$ and unable to walk $(\mathrm{n}=75) .6$-MWT in able to walk group was 218 meters (range: 128 - 297).35

\section{NT-proBNP}

Identification of at-risk groups is extremely important as early diagnosis and treatment can help prevent morbidity and mortality in $\mathrm{HF}^{3}$ Natriuretic peptides like BNP (B-type natriuretic peptide) and NT-proBNP (N-terminal pro-BNP) are produced predominantly in the left ventricle of the heart and released into the circulation in pressure or volume overload conditions. NT-proBNP is recently being used as a test for rapidly diagnosing and identifying the severity of HF in a patient with dyspnoea. Serial natriuretic peptides testing is useful in determining the efficacy of treatment of HF patients as well as defining prognosis in patients of HF. ${ }^{36-38}$

All-cause mortality is very high in patients with acute HF whose NT-proBNP levels are elevated at the time of admission. It is a reliable and powerful mortality predictor at 30-day and 1 -year after admission. The optimal cutoff level of NT-proBNP used to predict 30-day and 1-year mortality has a very high specificity and sensitivity. ${ }^{39}$ NT-proBNP level is considered to be a useful marker for risk stratification in acute HF. 40

Zile MR et al. analysed data from PARADIGM-HF to study the effect of the reduction of NT-proBNP on mortality rates. They concluded that patients attaining a significant reduction in NT-proBNP levels had lower hospitalization rates and lower mortality rates. This study holds prime importance as it suggests that NT-proBNP is directly associated with hospitalisation or cardiovascular mortality in HFrEF patients. The study also reveals that ARNI can help reduce NT-proBNP levels thereby improving outcomes in HFrEF.41

Domenico Scrutinio et al. assessed the relation of the updated ADHF / NT-proBNP risk score with ninety-day and inhospital deaths in 701 patients with advanced HF. The mean NT-proBNP levels in there study was 5,418 (range: 2,501 10,633). NT-proBNP of $>5180 \mathrm{pg} / \mathrm{ml}$ was found in 362 (51.4 $\%)$ out of total study population $(n=701)$, values above which had higher mortality rates. ${ }^{42}$

Claus Luers et al studied NT-proBNP plasma levels of 85 patients with decompensated HF. The levels were measured on admission and at $12 \mathrm{~h}$ after hospital admission. 26 patients (31\%) died within the first 30-days. NT-proBNP on admission among survivors was 4227 [1220, 8079] and among nonsurvivors was $7939[1879,12,499] .43$

\section{HbA 1c}

Diabetes and HF are two chronic diseases that usually coexist. The prevalence of diabetes is as high as $40 \% 44$ and this is on increasing trends Among HF patients. ${ }^{45}$ Diabetes in HF holds clinical importance as diabetes has been linked with worse outcomes. There remains uncertainty over the optimal HbA1c level for patients with HF. It is observed that higher levels of HbA1c have a direct association with increased hospitalization rates in HF patient. ${ }^{46}$ Recent interventional trials in diabetic patients have not shown any benefit of tight glycaemic control. Few studies have suggested harm more than good. ${ }^{47}$

David Aguilar, MD et al. studied the relationship between HbA1C and poor outcomes in diabetic patients with established HF. They proposed that tight glycaemic control predisposes to the retention of fluids in the body by reducing the osmotic diuretic effect of glucose, predisposing to HF. Moderate glycaemic control seems very appropriate as tight and loose glycaemic control both precipitates heart failure. The study was primarily done in male patients from veteran's affairs medical centres. They found a U-shaped relationship between serum HbA1c levels and mortality rates. The lowest risk was seen for patients with $\mathrm{HbA} 1 \mathrm{c}$ levels ranging between 7.1 and $7.8 \% .48$

Douglas H.J. Elder et al. performed a cohort study to corelate Mean HbA1c and mortality in diabetic individuals with HF. They had divided the cohort into 5 groups: HbA1c $<6.0 \%$, HbA1c 6.1 - $7.0 \%$, HbA1c 7.1 - $8.0 \%$, HbA1c 8.1 - $9.0 \%$ and HbA1c $>9.0 \%$. A Cox regression model, adjusted for all significant predictors, with the middle category $(7.1-8.0 \%)$ as the reference value, demonstrated a U-shaped association between $\mathrm{HbA1c}$ and outcome which is similar to our study 49

Ivan Cavero-Redondo et al performed an analytical study to examine the relationship between $\mathrm{HbA1c}$ levels and the outcomes depending on data from various studies and also to define the optimal levels of HbA1c for preventing worse cardiac outcomes in diabetic and non-diabetic populations. 46 studies were studied in this meta-analysis. There was an increase in mortality rates when HbA1c levels were above 8.0 $\%$ and $6.0 \%$, in diabetic and non-diabetic subjects respectively. However, all-cause mortality was also higher when $\mathrm{HbA} 1 \mathrm{c}$ was below $6.0 \%$ and below $5.0 \%$ in both diabetic and non-diabetic study groups respectively. It is a reliable marker of mortality in both groups. By the study, they tried to establish an optimal HbA1c level, for the lowest cardiovascular and all-cause mortality. ${ }^{50}$

\section{CONCLUSIONS}

The prevalence of HF is increasing all over the world. Early identification of risk factors can lead to early intervention and improve the prognosis of such individuals who are at higher risk of adverse events. In higher setup, the use of implantable devices, heart transplantation, and newer drug therapy have revolutionized the management and outcome of HF. However, its availability is limited. Various tests are used for determining the diagnosis and prognosis in advanced heart failure. The above mentioned are the few most commonly used ones. Recent studies have proved that NT-proBNP and 6-MWT have very good prognostic values. New markers are still under trial. Advanced heart failure is a very fatal condition and its treatment demands a multidisciplinary approach by the cardiologist, intensivist, and emergency medicine doctors.

Financial or other competing interests: None.

Disclosure forms provided by the authors are available with the full text of this article at jemds.com. 


\section{REFERENCES}

[1] Malik A, Brito D, Chhabra L. Congestive Heart Failure (CHF). In: StatPearls Tresure Island (FL): StatPearls Publishing 2020 Jan. 2020 Aug 10.

[2] Pereira-Barretto AC, de Oliveira MT, Strunz CC, et al. Serum NT-proBNP levels are a prognostic predictor in patients with advanced heart failure. Arq Bras Cardiol 2006;87(2):174-7.

[3] Redfield MM. Heart failure--an epidemic of uncertain proportions. N Engl J Med 2002;347(18):1442-4.

[4] Abouezzeddine OF, Redfield MM. Who has advanced heart failure? Definition and epidemiology. Congest Heart Fail 2011;17(4):160-8.

[5] Lip GYH, Gibbs CR, Beevers DG. Aetiology. BM] 2000;320(7227):104-7.

[6] Chaturvedi V, Parakh N, Seth S, et al. Heart failure in India: The INDUS (INDia Ukieri Study) study. J Pract of Cardiovasc Sci 2016;2(1):28-35. http://www.jpcs.org/article.asp?issn=2395-

5414 ; year $=2016$; volume $=2 ;$ issue $=1 ;$ spage $=28$; epage $=35$; aulast=Chaturvedi

[7] Costanzo MR, Mills RM, Wynne J. Characteristics of "Stage D" heart failure: insights from the Acute Decompensated Heart Failure National Registry Longitudinal Module (ADHERE LM). Am Heart J 2008;155(2):339-47.

[8] Crespo-Leiro MG, Metra M, Lund LH, et al. Advanced heart failure: a position statement of the Heart Failure Association of the European Society of Cardiology. Eur J Heart Fail 2018;20(11):1505-35.

[9] Wong M, Staszewsky L, Latini R, et al. Severity of left ventricular remodeling defines outcomes and response to therapy in heart failure: Valsartan heart failure trial (ValHeFT) echocardiographic data. J Am Coll Cardiol 2004;43(11):2022-7.

[10] Yoshihisa A, Sato Y, Kanno Y, et al. Prognostic impacts of changes in left ventricular ejection fraction in heart failure patients with preserved left ventricular ejection fraction. Open Heart 2020;7(1):e001112.

[11] Wong M, Staszewsky L, Latini R, et al. Valsartan benefits left ventricular structure and function in heart failure: Val-HeFT echocardiographic study. J Am Coll Cardiol 2002;40(5):970-5.

[12] Lam CSP, Teng T-HK, Tay WT, et al. Regional and ethnic differences among patients with heart failure in Asia: the Asian sudden cardiac death in heart failure registry. Eur Heart J 2016;37(41):3141-53.

[13] Konstam MA. Renal function and heart failure treatment. Circ Heart Fail 2011;4(6):677-9.

[14] Dries DL, Exner DV, Domanski MJ, et al. The prognostic implications of renal insufficiency in asymptomatic and symptomatic patients with left ventricular systolic dysfunction. J Am Coll Cardiol 2000;35(3):681-9.

[15] Hillege HL, Girbes AR, de Kam PJ, et al. Renal function, neurohormonal activation, and survival in patients with chronic heart failure. Circulation 2000;102(2):203-10.

[16] Damman K, Testani JM. The kidney in heart failure: an update. Eur Heart J 2015;36(23):1437-44.

[17] Testani JM, Kimmel SE, Dries DL, et al. Prognostic importance of early worsening renal function after initiation of angiotensin-converting enzyme inhibitor therapy in patients with cardiac dysfunction. Circ Heart Fail 2011;4(6):685-91.

[18] Chopra VK, Mittal S, Bansal M, et al. Clinical profile and one-year survival of patients with heart failure with reduced ejection fraction: The largest report from India. Indian Heart J 2019;71(3):242-8.

[19] Dewan P, Jhund PS, Shen L, et al. Heart failure with reduced ejection fraction: comparison of patient characteristics and clinical outcomes within Asia and between Asia, Europe and the Americas. Eur J Heart Fail 2019;21(5):577-87.

[20] Gheorghiade M, Abraham WT, Albert NM, et al. Relationship between admission serum sodium concentration and clinical outcomes in patients hospitalized for heart failure: an analysis from the OPTIMIZE-HF registry. Eur Heart J 2007;28(8):980-8.

[21] Felker GM, Leimberger JD, Califf RM, et al. Risk stratification after hospitalization for decompensated heart failure. J Card Fail 2004;10(6):460-6.

[22] Gheorghiade M, Gattis WA, O'Connor CM, et al. Effects of tolvaptan, a vasopressin antagonist, in patients hospitalized with worsening heart failure: a randomized controlled trial. JAMA 2004;291(16):1963-71.

[23] Kearney MT, Fox KAA, Lee AJ, et al. Predicting death due to progressive heart failure in patients with mild-tomoderate chronic heart failure. J Am Coll Cardiol 2002;40(10):1801-8.

[24] Gheorghiade M, Rossi JS, Cotts W, et al. Characterization and prognostic value of persistent hyponatremia in patients with severe heart failure in the ESCAPE Trial. Arch Intern Med 2007;167(18):1998-2005.

[25] Corona G, Giuliani C, Parenti G, et al. Moderate hyponatremia is associated with increased risk of mortality: evidence from a meta-analysis. PLoS One 2013;8(12):e80451.

[26] Mohammed AA, van Kimmenade RRJ, Richards M, et al. Hyponatremia, natriuretic peptides and outcomes in acutely decompensated heart failure: results from the International Collaborative of NT-proBNP Study. Circ Heart Fail 2010;3(3):354-61.

[27] Kervio G, Ville NS, Leclercq C, et al. Intensity and daily reliability of the six-minute walk test in moderate chronic heart failure patients. Arch Phys Med Rehabil 2004;85(9):1513-8.

[28] Owan TE, Hodge DO, Herges RM, et al. Trends in prevalence and outcome of heart failure with preserved ejection fraction. N Engl J Med 2006;355(3):251-9.

[29] Larsen AI, Aarsland T, Kristiansen M, et al. Assessing the effect of exercise training in men with heart failure; comparison of maximal, submaximal and endurance exercise protocols. Eur Heart J 2001;22(8):684-92.

[30] Pepera GK, Sandercock GR, Sloan R, et al. Influence of step length on 6-minute walk test performance in patients with chronic heart failure. Physiotherapy 2012;98(4):325-9.

[31] Alahdab MT, Mansour IN, Napan S, et al. Six minute walk test predicts long-term all-cause mortality and heart failure rehospitalization in African-American patients hospitalized with acute decompensated heart failure. J Card Fail 2009;15(2):130-5. 
[32] Rubim VSM, Drumond Neto C, Romeo JLM, et al. Prognostic value of the six-minute walk test in heart failure. Arq Bras Cardiol 2006;86(2):120-5.

[33] Wegrzynowska-Teodorczyk K, Rudzinska E, Lazorczyk M, et al. Distance covered during a six-minute walk test predicts long-term cardiovascular mortality and hospitalisation rates in men with systolic heart failure: an observational study. J Physiother 2013;59(3):177-87.

[34] Cahalin LP, Mathier MA, Semigran MJ, et al. The sixminute walk test predicts peak oxygen uptake and survival in patients with advanced heart failure. Chest 1996;110(2):325-32.

[35] Shah MR, Hasselblad V, Gheorghiade M, et al. Prognostic usefulness of the six-minute walk in patients with advanced congestive heart failure secondary to ischemic or nonischemic cardiomyopathy. Am J Cardiol 2001;88(9):987-93.

[36] Trinquart L, Ray P, Riou B, et al. Natriuretic peptide testing in EDs for managing acute dyspnea: a metaanalysis. Am J Emerg Med 2011;29(7):757-67.

[37] Carpenter CR, Keim SM, Worster A, et al. BEEM (Best Evidence in Emergency Medicine). Brain natriuretic peptide in the evaluation of emergency department dyspnea: Is there a role? J Emerg Med 2012;42(2):197205.

[38] Januzzi JL, van Kimmenade R, Lainchbury J, et al. NTproBNP testing for diagnosis and short-term prognosis in acute destabilized heart failure: an international pooled analysis of 1256 patients: the International Collaborative of NT-proBNP Study. Eur Heart J 2006;27(3):330-7.

[39] Golcuk Y, Golcuk B, Velibey Y, et al. Predictive cutoff point of admission $\mathrm{N}$-terminal pro-B-type natriuretic peptide testing in the ED for prognosis of patients with acute heart failure. Am J Emerg Med 2013;31(8):1191-5.

[40] Palazzuoli A, Gallotta M, Quatrini I, et al. Natriuretic peptides (BNP and NT-proBNP): measurement and relevance in heart failure. Vasc Health Risk Manag 2010;6:411-8.

[41] Zile MR, Claggett BL, Presscott MF, et al. NT-proBNP as a Prognostic Marker in Heart Failure. American College of Cardiology 2016;68:2425-36. http\%3a\%2f\%2fwww.acc.org\%2flatest-incardiology\%2fjournal- scans $\% 2 \mathrm{f} 2016 \% 2 \mathrm{f} 11 \% 2 \mathrm{f} 29 \% 2 \mathrm{f} 12 \% 2 \mathrm{f} 15 \% 2$ fprognosticimplications-of-changes-in-ntprobnp

[42] Scrutinio D, Ammirati E, Passantino A, et al. Predicting short-term mortality in advanced decompensated heart failure - role of the updated acute decompensated heart failure/N-terminal pro-B-type natriuretic Peptide risk score. Circ J 2015;79(5):1076-83.

[43] Luers C, Sutcliffe A, Binder L, et al. NT-proANP and NTproBNP as prognostic markers in patients with acute decompensated heart failure of different etiologies. Clin Biochem 2013;46(12):1013-9.

[44] Adams KF Jr, Fonarow GC, Emerman CL, et al. Characteristics and outcomes of patients hospitalized for heart failure in the United States: rationale, design, and preliminary observations from the first 100,000 cases in the Acute Decompensated Heart Failure National Registry (ADHERE). Am Heart J 2005;149(2):209-16.

[45] From AM, Leibson CL, Bursi F, et al. Diabetes in heart failure: prevalence and impact on outcome in the population. Am J Med 2006;119(7):591-9.

[46] Romero SP, Garcia-Egido A, Escobar MA, et al. Impact of new-onset diabetes mellitus and glycaemic control on the prognosis of heart failure patients: a propensity-matched study in the community. Int J Cardiol 2013;167(4):120616.

[47] Duckworth W, Abraira C, Moritz T, et al. Glucose control and vascular complications in veterans with type 2 diabetes. N Engl J Med 2009;360(2):129-39.

[48] Aguilar D, Bozkurt B, Ramasubbu K, et al. Relationship of Hemoglobin A1c and mortality in heart failure patients with diabetes. J Am Coll Cardiol 2009;54(5):422-8.

[49] Elder DHJ, Singh JSS, Levin D, et al. Mean HbA1c and mortality in diabetic individuals with heart failure: a population cohort study. Eur J Heart Fail 2016;18(1):94102.

[50] Cavero-Redondo I, Peleteiro B, Álvarez-Bueno C, et al. Glycated haemoglobin $\mathrm{A} 1 \mathrm{C}$ as a risk factor of cardiovascular outcomes and all-cause mortality in diabetic and non-diabetic populations: a systematic review and meta-analysis. BMJ Open 2017;7(7):e015949. 